International Journal of Pure and Applied Mathematics

Volume 93 No. 4 2014, 581-602

ISSN: 1311-8080 (printed version); ISSN: 1314-3395 (on-line version)

url: http://www.ijpam.eu

doi: http://dx.doi.org/10.12732/ijpam.v93i4.8

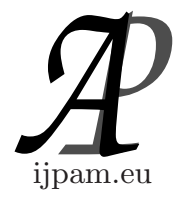

\title{
CRITICAL SLOWING DOWN IN BIOLOGICAL BISTABLE MODELS
}

\author{
R.A. Alharbey ${ }^{1 \S}$, L.A.M. Nejad ${ }^{2}$, S. Lynch ${ }^{2}$, S.S. Hassan ${ }^{2,3}$ \\ ${ }^{1}$ Mathematics Department \\ Faculty of Science \\ King Abdul-Aziz University \\ P.O. Box 42696, Jeddah, 21551, KINGDOM OF SAUDI ARABIA \\ ${ }^{2}$ School of Computing, Maths. \& Digital Technology \\ Manchester Metropolitan University \\ Manchester M1 5GD, UK \\ ${ }^{3}$ Department of Mathematics \\ College of Science \\ University of Bahrain \\ P.O. Box 32038, KINGDOM OF BAHRAIN
}

\begin{abstract}
We investigate both analytically and computationally, the bistable behaviour in some biological mathematical models, namely: spruce budworm infestations, the Thomas reaction model and the activator-inhibitor model of Gierer-Meinhardt.

Bistable behaviour is achieved by treating some of the concentration, supply, degradation and reaction rates as control parameters. Critical slowing down in the switching time response due to perturbed control parameters is examined at the critical points of the bistable curves. Switching between stable states may occur sharply, monotonically, non-monotonically or via oscillations, depending on the nonlinear feedback process. The scaling for the switching delay time fits the inverse square root law $\beta^{-\frac{1}{2}}$ for the three models ( $\beta$ is the perturbation of the control parameter).
\end{abstract}

Key Words: bistable behaviour, critical slowing down, mathematical models in biology

Received: March 26, 2014

(C) 2014 Academic Publications, Ltd.

$\S_{\text {Correspondence author }}$ url: www.acadpubl.eu 


\section{Introduction}

Bistable behaviour in a physical system is a co-existence of two stable (output) states due to a single control (input) parameter. This nonlinear behaviour has been extensively studied, both theoretically and experimentally in the field of nonlinear optics since the 1970's (e.g. [1]-[6] and more recently in [7] and [8]) and still plays a central role in recent research topics. For example, optical computers and optical devices that can be manipulated to reach super- and subluminal group velocity ([9], [10] and refs. therein). Furthermore, the transient switching response in optical bistable systems has been examined in [11-13] where the switching time from lower to upper state (or vice-versa) diverges near the critical switching-up or -down points. This phenomenon is called Critical Slowing Down (CSD). The effect of perturbation (or fluctuation) of the input field at the critical points shows that CSD can be utilised to achieve dynamical stabilisation of the bistable system [14]. Also, feasibility of device application using the CSD effect in optics was reported in [15] and [16].

Theory and observation show that real biological and ecological systems exhibit bi- and multi-stable behaviour [17] and [18] in a similar fashion to optical systems. Further specific examples are the following: the demonstration of bistable behaviour in neuromodules containing one and two neurones [19]; analysis of blood cell population models that show cell populations are subject to hysteresis behaviour [20]; demonstration of bistable behaviour in some versions of enzyme membrane and activator-inhibitor reaction models [21]; and finally, the many physiological oscillatory processes in the human body that are subject to nonlinearity, feedback and hysteresis behaviour [22].

The work in this paper is concerned with the following three biological models:

(i) the spruce budworm outbreak model [23];

(ii) the Thomas reaction (enzyme membrane) model [24]; and

(iii) the activator-inhibitor reaction model of biological pattern formation by Gierer and Meinhardt (GM) [25].

The main objectives of the present work regarding the above three models are three fold: The first, is to investigate the possibility of additional bistable behaviour and its regions through treating some of the system characteristic rates as control parameters. The second, is to examine the CSD phenomenon as a result of small perturbations of the input (control) parameter near the vicinity of the critical points of the bistable curve, specifically, the nature of 
transition between the stable states. The third is the verification of the inverse square root scaling law for the switching time delay in the vicinity of the critical (switching- on and -off) points, independent of the nonlinearity in the three models.

The paper is presented as follows: The model equations together with the identification of the bistable regions, investigation of the transient switching and its time delay for the three biological models; the spruce budworm, Thomas and GM reaction models are presented in Sections 2 - 4, respectively. A summary is given in Section 5 .

\section{The Spruce Budworm Outbreak Model}

As discussed by May [17], this mathematical model applies to other ecological systems, such as grazing systems and some human-host parasite systems. For our concern, the spruce budworm model provides a good example to understand the dynamics of the interacting ecosystem of trees and insects [26]. It describes the oscillatory behaviour of the spruce budworm outbreak of infestations in forests, which in particular defoliate balsam fir trees.

The main features to be considered in this model are:

(i) leaves of the trees provide food as well coverage for budworms (to hide from birds);

(ii) birds feed on the budworms (if they are in large supply) and other food resources. Therefore, the bird populations are taken to be constant.

The model rate equation for the budworm population to be considered here has the form [23] and [26]:

$$
\frac{d N}{d \tau}=N\left[\left(1-\frac{N}{K}\right)-\frac{F N}{N^{2}+B^{2}}\right] .
$$

The parameters in (1) are as follows: $N$ is the budworm population, $\tau=r t$ is the normalised time and $r$ is the linear birth rate of the budworm, $K$ is the constant carrying capacity, which is related to the density of foliage (food) available on the trees in the absence of birds. The term $\frac{F N}{N^{2}+B^{2}}$, represents predation generated by birds with $F=\frac{p_{o} A}{r}$, being the predation population ( $p_{o}$ is the constant predation rate, $A$ is a positive constant representing the predator attack rate) and $B$ is a measure of the threshold for the budworm population. 
(i) Bistable regions In the steady state, $\frac{d N}{d \tau}=0$, and treating the parameter $K$ as a control parameter and solving for $K$, the characteristic equations between $K$ and $N$ have been obtained, with $F$ and $B$ taken positive constants [17], [18], [23] and [26],

$$
\begin{aligned}
K & =N\left(1+\frac{F N}{N^{2}+B^{2}-F N}\right) . \\
& =\frac{N^{3}+B^{2} N}{N^{2}+B^{2}-F N} .
\end{aligned}
$$

The right side of $(2 \mathrm{~b})$ is a rational function with a cubic polynomial numerator in $N$ and the conditions for bistable behaviour are (cf. [5]),

$$
N_{i n f}>0 \text { and }\left.S_{o}(F, B) \equiv \frac{d K}{d N}\right|_{N_{i n f}} \leq 0
$$

where $N_{\text {inf }}$ is the inflection point obtained by solving $\frac{d^{2} K}{d N^{2}}=0$, and choosing the positive real root of $N$.

The search for the region of bistability is via the isolines that satisfy the second condition in (3) which defines a surface function $S_{o}(F, B)$ in the two parameters $F$ and $B$ (see inset in Fig.1). Regions of bistability (shaded area) exist for increasing $F$ and $B$ such that: $0<F<1,0<$ $B \leq 0.5$, Fig.1(a). For fixed $B=0.5, F=0.85$ steady state pronounced bistable curve ( $N$ against $K$ ) according to (2) is shown in Fig.1(b) with $A_{o n}, A_{o f f}$ being the switching-on and -off points, respectively.

(ii) Transient switching and delay Here, we investigate the switching time from upper to lower branch (or vice-versa) of the bistable curve due to positive or negative linear perturbation of the control (input) parameter, $K_{c} \pm \beta$ where $0<\beta<1$ is a (real) small perturbation and $K_{c}$ is the value of $K$ at the critical switching points. Using Runge-Kutta-Fehlberg method and Maple 15, we numerically integrate (1) with steady state values for the initial conditions (IC) and fixed values of $B=0.5$ and $F=0.85$, (Fig.1(b)).

At the switching-on point with positive perturbation (i.e after crossing the threshold), $A_{\text {on }}=(3.6631+\beta, 0.61299)$, the transient behaviour of $N(\tau)$ for various perturbation $\beta$ is shown in Fig.2a. For small $\beta=10^{-3}$ (nearer to the critical point $A_{\text {on }}$ ) there is a divergence (slowing down) 

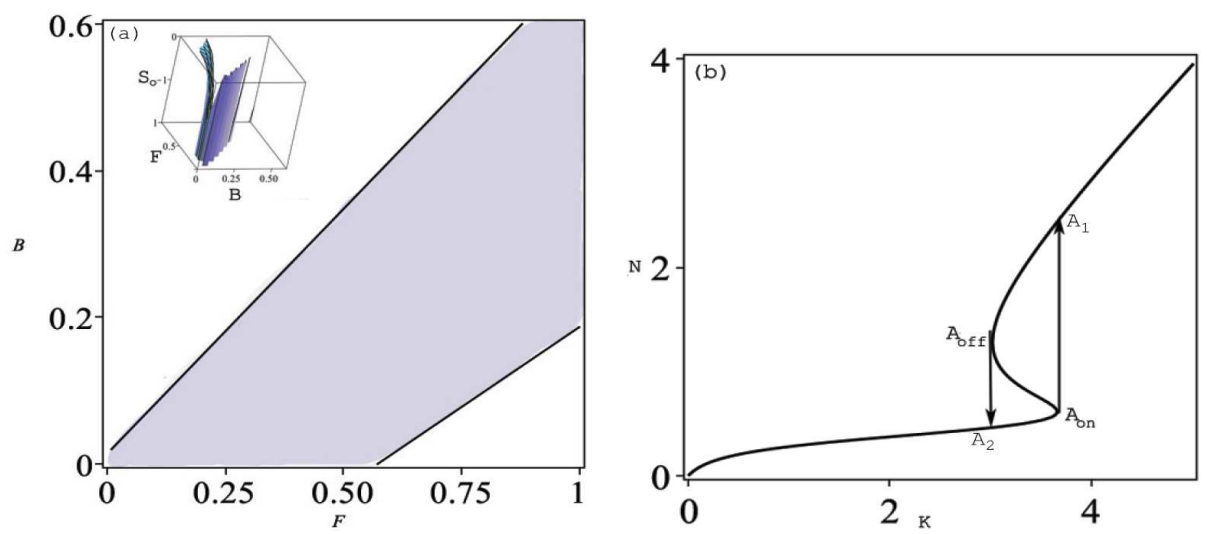

Fig.1: (a) Bistable region for the population $N$ in the $(F, B)$-plane. Inset shows the $3 \mathrm{D}$ plot of the surface $S_{o}(F, B)$, eq.(3), as function of the two parameters $F, B$. (b) Steady state bistable curve of eq.(2), $N$ against $\mathrm{K}$, at fixed values of $F=0.85, B=0.5$. The switching-on and -off points: $A_{o n}=(3.6631,0.61299)$ and $A_{o f f}=(3.0199,1.2793)$ with terminal points $A_{1}=(3.6631,2.4371)$ and $A_{2}=(3.0199,0.46129)$, respectively.

to switch-up to the steady upper-branch $(N \sim 2.5)$. Increasing $\beta$ (i.e. shifting away to the right from point $A_{o n}$ ) tends to reduce the time delay to switch-up to the steady state value of $N$.

For negative perturbation (i.e before crossing the threshold $A_{\text {on }}$ ), $A_{\text {on }}=$ $(3.6631-\beta, 0.61299)$, the steady state reached corresponds to the lower steady state branch $(N \sim 0.6)$, Fig.2b. For small $\beta=10^{-3}$, at the switching point $A_{\text {on }}$, Fig.2c shows the sharp convergence to the upper branch of the hysteresis curve (curve $C_{a}$ after crossing the threshold), compared with slower convergence to the lower branch of the hysteresis curve, (curve $C_{b}$ before crossing the threshold).

At the switching-off point with positive perturbation (before crossing the threshold $), A_{o f f}=(3.0199+\beta, 1.2793)$, the transient behaviour of $N(t)$ is shown in Fig.3a. For small $\beta=10^{-3}$ the divergence to switch-up to very near upper steady state $(N=1.28 \rightarrow 1.31)$ occurred smoothly compared with Fig.2a. For negative perturbation (after crossing the threshold), $A_{\text {off }}=(3.0199-\beta, 1.2793)$, Fig. $3 \mathrm{~b}$, and for $\beta=10^{-3}$ the reach to the lower steady state of the bistable curve $(N \sim 0.46)$ is sharp compared with Fig.3a. Fig.3c for small $\beta=10^{-3}$ at $A_{o f f}$, shows the opposite behaviour 

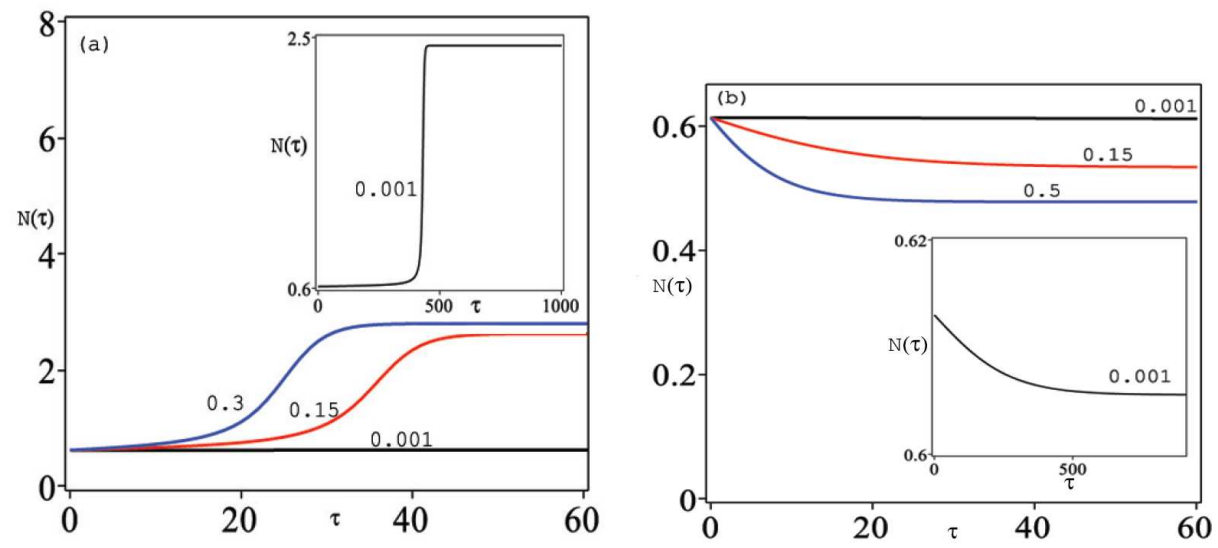

Fig.2: (a) The transient population $N(\tau)$ against the normalised time $\tau=r t$ for $F=0.85, B=0.5$ and various values of the control parameter with positive perturbation, $K=K_{c}+\beta$ (after threshold); $K_{c}=3.6631$ (at the switching-on point $A_{o n}$ in Fig.1(b)) and $\beta=0.001,0.15$ and 0.3 . Inset shows $N(\tau)$ for the smallest perturbation $(\beta)$ near the threshold point $A_{o n}$. (b) As (a) but with negative perturbation, $K=K_{c}-\beta$ (before threshold) and $\beta=0.001,0.15$ and 0.5 .

of Fig.2c at $A_{\text {on }}$, for the convergence to reach the steady state branches of the bistable curve.

To examine the behaviour of the time-delay $\tau_{d}$ to reach either upper or lower steady state of the hysteresis curve against the perturbation $(\beta)$ of the control parameter $(K)$, we numerically integrate Eq.(1) over continuous interval of $\beta \in(0,0.3)$ to calculate $\tau_{d}$. For fixed $B=0.5, F=$ 0.85 the results in Fig.4 are essentially the same for positive or negative perturbation at either critical point $A_{\text {on }}$ or $A_{\text {off }}$ and show that $\tau_{d} \sim \beta^{-\frac{1}{2}}$, i.e proportional to the inverse square root scaling law.

\section{The Thomas Reaction Model}

Within the artificial enzyme membrane of diffusion cells [24], the membrane separates in to two compartments containing solutions of substrates (product and effector). Both solutions enter the membrane as "inputs" and the output solution flux is then analysed. A specific Thomas reaction model for enzyme membrane [18] is that which involves two substrates, Oxygen and Uric acid, of 


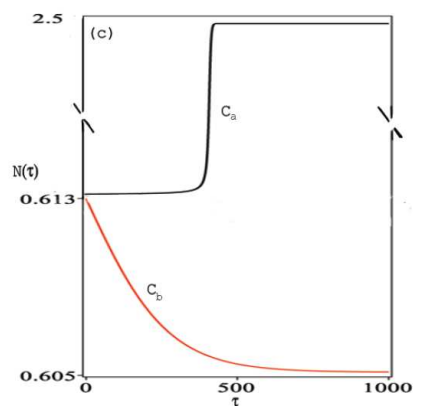

Fig.2: (c) As Fig.2(a) for positive and negative perturbation, $K=$ $K_{c} \pm \beta$, curves $C_{a}$ and $C_{b}$ (after and before threshold), respectively, with $K_{c}=3.6631$ (at the switching-on point $A_{\text {on }}$ in Fig.1(b)) and $\beta=0.001$.
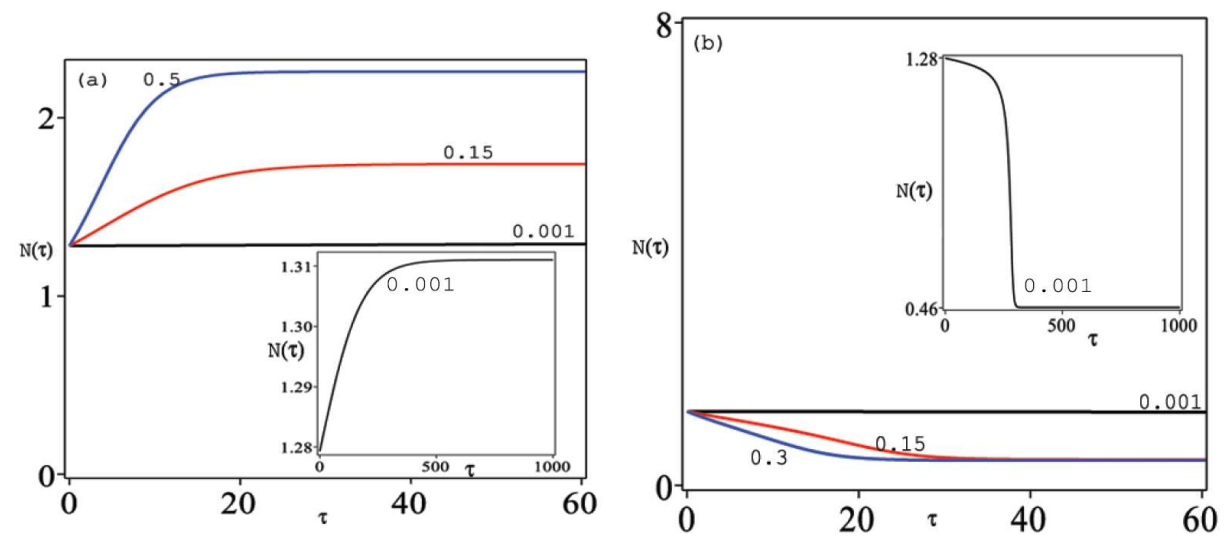

Fig.3: (a) As Fig.2(a) for positive perturbation, $K=K_{c}+\beta$ (before threshold) but with $K_{c}=3.0199$ (at the switching-off point $A_{\text {off }}$ in Fig.1(b)) and $\beta=0.001,0.15$ and 0.5. Inset shows $N(\tau)$ for the smallest perturbation $(\beta)$ near the threshold point $A_{\text {off }}$. (b) As (a) but with negative perturbation, $K=K_{c}-\beta$ (after threshold) and $\beta=0.001,0.15$ and 0.3 .

concentrations $u$ and $v$, respectively. The rate equations for $u$ and $v$ are of the following dimensionless form [18],

$$
\begin{aligned}
& \dot{u}=a-u-l \frac{u v}{1+u+k u^{2}} \\
& \dot{v}=\alpha(b-v)-l \frac{u v}{1+u+k u^{2}},
\end{aligned}
$$




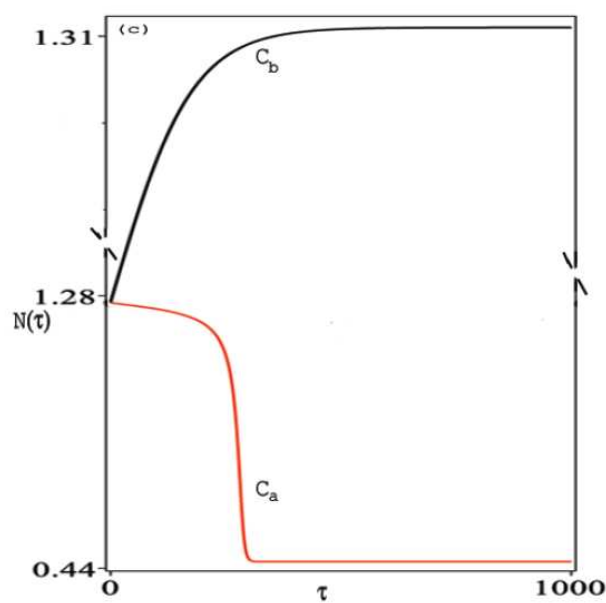

Fig.3: (c) $N(\tau)$ versus $\tau$ with positive and negative perturbation, $K=$ $K_{c} \pm \beta$, curves $C_{b}$ and $C_{a}$ (before and after threshold), respectively, with $K_{c}=3.0199$ (at the switching-off point $A_{o f f}$ in Fig.1b) and $\beta=0.001$.

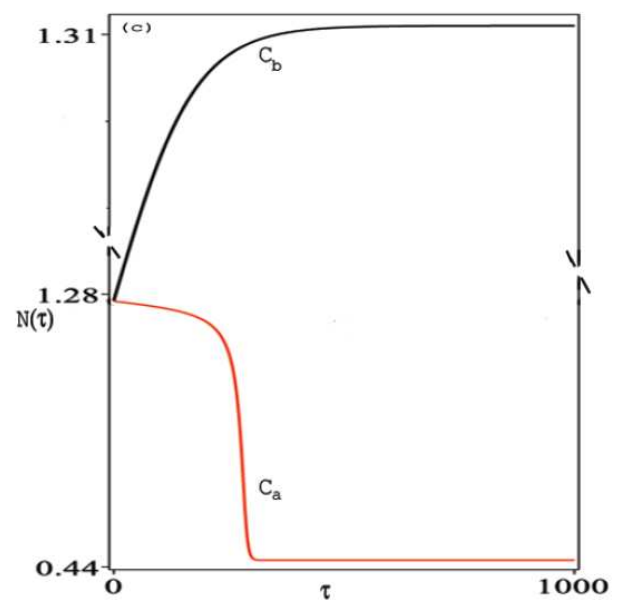

Fig.4: Time-delay $\tau_{d}$ against the perturbation $(\beta)$, black curve. The scaling law $\tau_{d}=\tau_{o} \beta^{-\frac{1}{2}}, \tau_{o}=23$ is represented by the red curve.

where all constants $a, b, \alpha, k$ and $l$ are positive real constants: $a$ and $\alpha b$ are supplied rates for $u$ and $v$, respectively. The terms $-u$ and $-\alpha v$ describe linear degradation processes, while the non-linear term $l$ is reaction rate (substrate inhibition) and $k$ is a measure of severity of inhibition. 
The steady state solutions $(\dot{u}=\dot{v}=0)$ of (4) gives the coupled state equations:

$$
\begin{aligned}
a & =u\left(1+\frac{l \alpha b}{\alpha+(l+\alpha) u+\alpha k u^{2}}\right), \\
v & =\frac{\alpha b\left(1+u+k u^{2}\right)}{\alpha+(l+\alpha) u+\alpha k u^{2}}, \\
& \equiv \frac{u-a}{\alpha}+b . \quad \text { (as well) }
\end{aligned}
$$

Next, we consider two cases:

(A) The supplied rate $a$ for the concentration $u$ as control parameter.

(i) Bistable regions Choosing $\alpha=l=1$ and hence from (5) the conditions for bistable behaviour in $u$ and $v$, i.e.

$$
\left.S_{1}(k, b) \equiv \frac{d a}{d u}\right|_{u_{i n f}} \leq 0 \quad \text { and }\left.\quad S_{2}(k, b) \equiv \frac{d a}{d v}\right|_{v_{i n f}} \leq 0,
$$

define the surfaces in the two parameters $(k, b)$.

Conditions (6) are treated computationally and show that for the $u$-concentration, the bistable behaviour is possible for $b, k \gtrsim 20,(b, k) \neq$ 0 , (Fig.5), while for the $v$-concentration, bistable behaviour is possible for all $b, k>0$.

At fixed values of $k=20, b=100, \alpha=l=1$ the concentration $u$ against $a$ exhibits the usual S-shape (Fig.6(a)), whilst the concentration $v$ against $a$ ( Fig.6(b)) starts at the fixed value of $b=100$ at $a=0$ (see eq. $(5 \mathrm{c})$ ), then decreases linearly until it switches up at point $A_{\text {on }}$.

(ii) Transient switching and delay The switching at the critical points $A_{o n}$ and $A_{o f f}$ in Fig.6 due to small perturbation of the control parameter $a \pm \beta$ is investigated by numerically integrated eq.(4) with the steady state values for $u, v$ as IC at fixed $k=20, b=100$, $\alpha=l=1$. Below, we show the results for $u(t)$ only, since $v(t)$ shows qualitatively similar behaviour.

At the switching-on point for $u(t)$ with positive perturbation in Fig.6(a), $A_{o n}=(9.3643+\beta, 0.2306)$ the transient behaviour of $u(t)$ is shown in Fig.7(a) for various $\beta$. For $\beta=10^{-4}$ divergence takes place until sharp switching-up occurs to the steady upper-branch near the point $A_{1}$ in Fig.6(a). For the same value of $\beta$ but with negative 


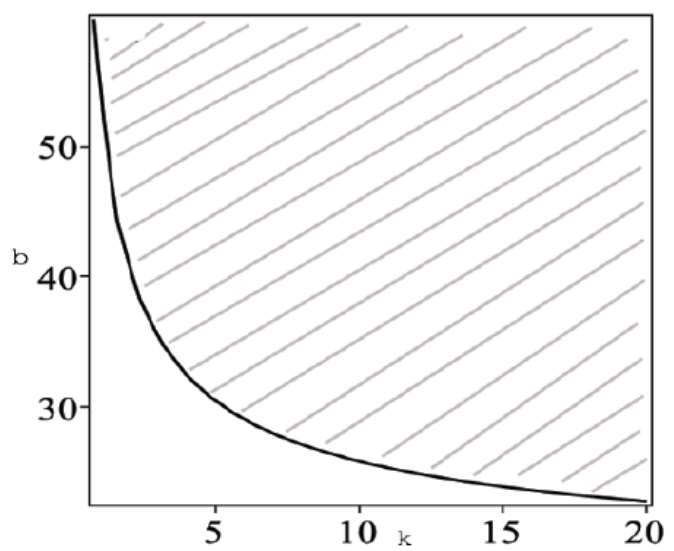

Fig.5: Bistable region for the concentration $u$ in the $(k, b)$-plane with $\alpha=l=1$.
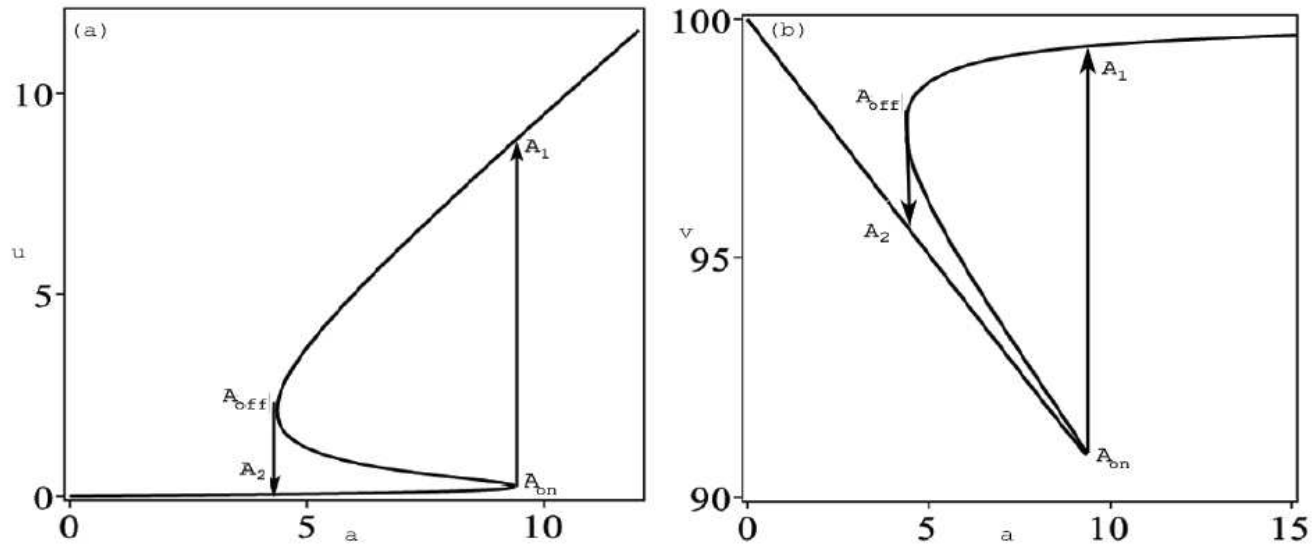

Fig.6: (a) Steady state bistable curve of eq.(5), $u$ against $a$, at fixed values of $k=20, b=100, \alpha=l=1$. The switching-on and -off points: $A_{\text {on }}=(9.3643,0.2306)$ and $A_{\text {off }}=(4.3484,2.0995)$ with terminal points $A_{1}=(9.3643,8.8030)$ and $A_{2}=(4.3484,0.04932)$, respectively. (b) As (a) but for the concentration $v$ against $a$. The switching-on and -off points: $A_{o n}=(9.3643,90.867)$ and $A_{o f f}=(4.3484,97.7511)$ with terminal points $A_{1}=(9.3643,99.4388)$ and $A_{2}=(4.3484,95.7009)$, respectively 

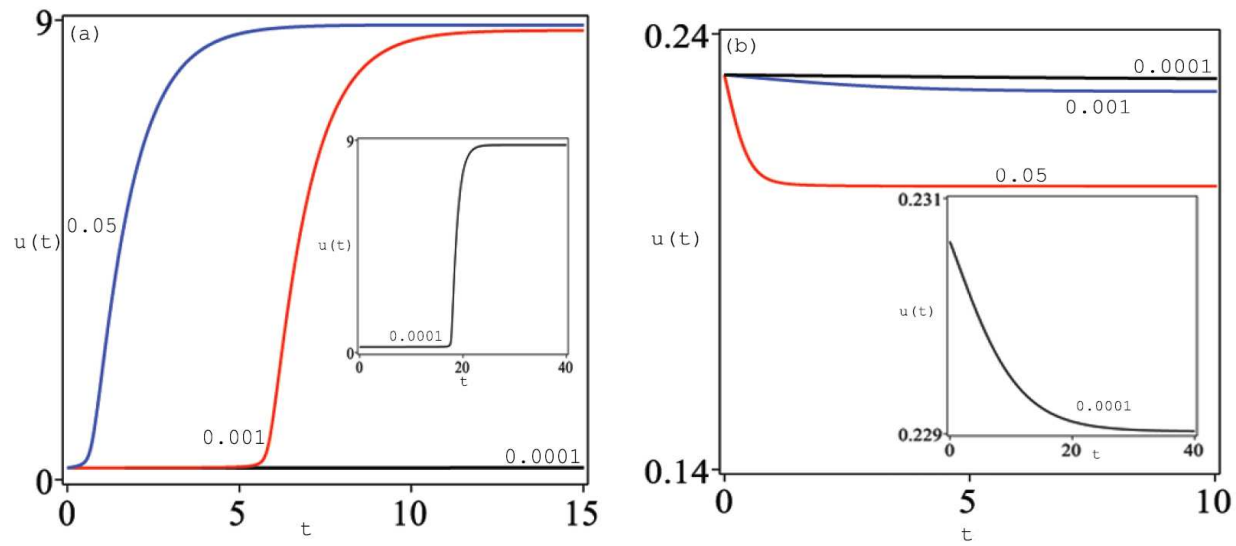

Fig.7: (a) The transient concentration $u(t)$ for $k=20, b=100, \alpha=$ $l=1$ and various values of the control parameter with positive perturbation, $a+\beta$ (after threshold); $a=9.3643$ (at the switching-on point $A_{\text {on }}$ in Fig.5) and $\beta=0.0001,0.001$ and 0.05. Inset shows $u(t)$ for the smallest perturbation $(\beta)$ near the threshold point $A_{\text {on }}$. (b) As (a) but with negative perturbation, $a-\beta$ (before threshold).

perturbation, Fig.7(b), $A_{o n}=(9.3643-\beta, 0.2306)$ there is a slight monotonic delay near the point $A_{o n}$. The comparison between the sharp switch-up and slow switch-down of $u(t)$, with smaller perturbation after and before crossing the threshold, respectively, is shown in Fig.7(c).

At the switching-off point $A_{\text {off }}$ in Fig.6(a), with positive perturbation, $A_{o f f}=(4.3484+\beta, 2.0995)$ the transient switching for $u(t)$ (Fig.8(a)), shows slow monotonic approach to a steady upper branch near the point $A_{\text {off }}$ (similar to Fig.7(b) with negative perturbation at the switching-up point $A_{o n}$ ).

Similarly, with negative perturbation at $A_{o f f}=(4.3484-\beta, 2.0995)$ the switching down to the steady lower branch with $u \sim 0.04932$ shows sharp behaviour (Fig.8(b)), (similar to Fig.7(a) with positive perturbation at the switching-on point $A_{o n}$ ). The slow and sharp switching to upper and lower steady branches with negative and positive small perturbation, respectively, is shown in-Fig.8(c).

The time-delay $\tau_{d}$ (for $u(t)$ or $v(t)$ ) against the perturbation parameter $\beta$ is shown in Fig.9: positive and negative $\beta$ show slight qualitative differ- 


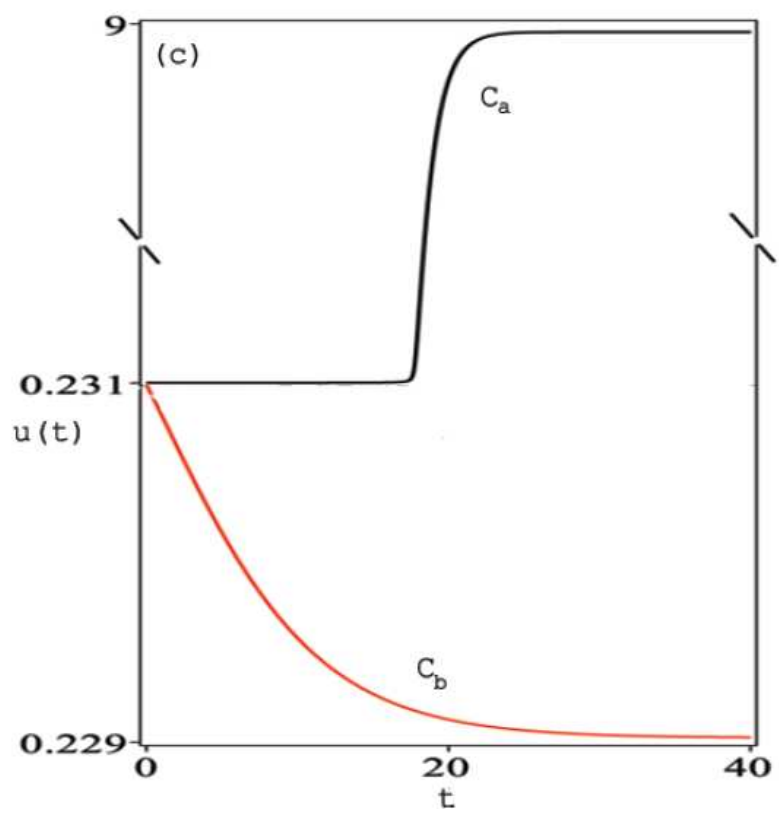

Fig.7: (c) $u(t)$ versus $t$ with positive and negative perturbation, $a \pm \beta$, curves $C_{a}$ and $C_{b}$ (after and before threshold), respectively, with $a=$ 9.3643 (at the switching-on point $A_{\text {on }}$ in Fig.5) and $\beta=0.0001$.

ence for $0<\beta \lesssim 0.1$ and in both cases $\tau_{d} \sim \beta^{-\frac{1}{2}}$.

(B) The effective supplied rate $b$ for the concentration $v$ as control parameter. From (5a), we have,

$$
b=\frac{\left(\frac{a}{u}-1\right)\left(\alpha+(l+\alpha) u+\alpha k u^{2}\right)}{l \alpha}
$$

whilst $b$ in terms of $v$ is given implicitly via $u$ from $(5 \mathrm{~b})$ or $(5 \mathrm{c})$.

For fixed $\alpha=\frac{1}{2}$ and $l=10$, the bistable regions in the $(k, a)-$ plane for $u$ and $v$ that satisfy the conditions,

$$
\left.S_{3}(k, a) \equiv \frac{d b}{d u}\right|_{u_{i n f}} \leq 0 \text { and }\left.S_{4}(k, a) \equiv \frac{d b}{d v}\right|_{v_{\text {inf }}} \leq 0,
$$

are $a k \gtrsim 20$ for $u$ and $a k \gtrsim 25$ for $v$ and both resemble qualitatively the shaded region in Fig.5 with axis-b replaced by axis-a. 

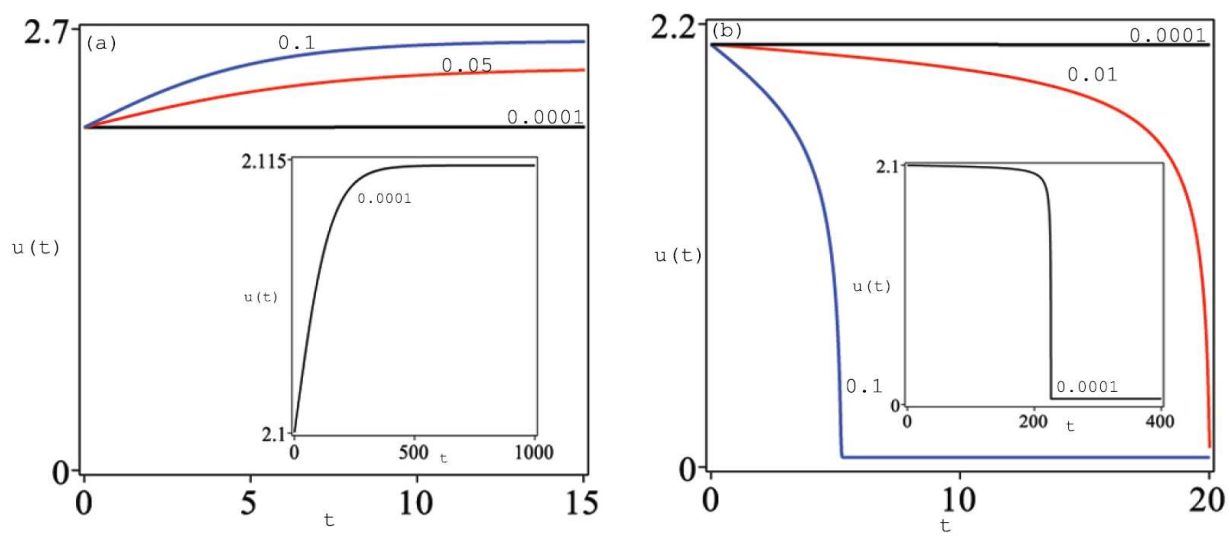

Fig.8: (a) The transient concentration $u(t)$ for $k=20, b=100, \alpha=$ $l=1$ and various values of the control parameter with positive perturbation, $a+\beta$ (after threshold); $a=4.3484$ (at the switching-off point $A_{\text {off }}$ in Fig.5) and $\beta=0.0001,0.05$ and 0.1 . Inset shows $u(t)$ for the smallest perturbation $(\beta)$ near the threshold point $A_{\text {off }}$. (b) As (a) but with negative perturbation, $a-\beta$ (before threshold) and $\beta=0.0001,0.01$ and 0.1 .

The bistable curves for the $u$ and $v$ against the control parameter $b$ Fig.10 show clockwise bistable shape, unlike the anti-clockwise loop in Fig.6 against the control parameter $a$.

The transient behaviour for $u$ and $v$ with positive or negative perturbations at the switching-on and off points in Fig.10 is basically the same as those in Figs. $(7,8)$ with $a$ as the control parameter. Likewise, for the time-delay $\tau_{d} \sim \beta^{-\frac{1}{2}}$ as in Fig.9.

\section{The Activator-Inhibitor (GM) Reaction Model}

Gierer and Meinhardt (GM) [25] have studied various models of biological pattern formation, especially the formation of a special (periodic or aperiodic) pattern of tissue structures. In one version of their models, namely, the morphogenetic pattern formation, the elementary process is considered as the formation of a primary pattern of two morphogene substrates: activator and inhibitor mutually interacting on their respective rates of production or degradation. The model is described by the coupled rate equations for the activator $u$ and in- 


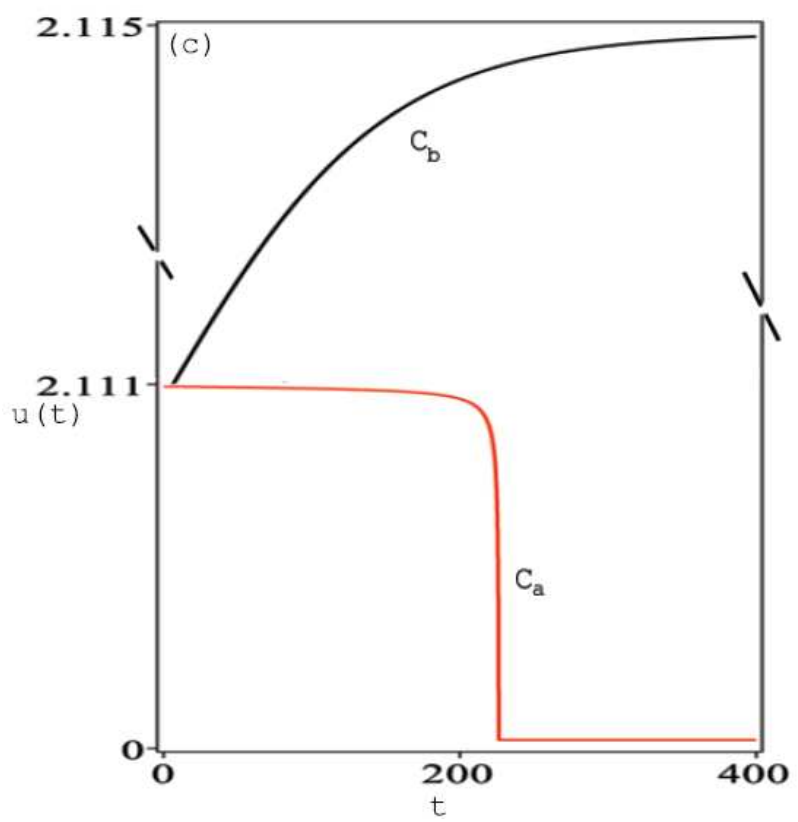

Fig.8: (c) $u(t)$ versus $t$ with positive and negative perturbation, $a \pm \beta$, curves $C_{b}$ and $C_{a}$ (before and after threshold), respectively, with $a=$ 4.3484 (at the switching-off point $A_{o f f}$ in Fig.5) and $\beta=0.0001$.

hibitor $v$ in dimensionless form [25],

$$
\begin{aligned}
& \dot{u}=a-b u+k u^{2} v \\
& \dot{v}=1-v-c u^{2} v,
\end{aligned}
$$

with $a, b, k$ and $c$ are positive real constants. The terms $-b u$ and $-v$ represent degradation processes, and the terms $a$ and 1 represent the basal production of activator and inhibitor, respectively. The nonlinear terms in $u^{2}$ in $(9 a, b)$ represent production and consumption processes proportional to $k, c$, respectively, and are due to an auto-catalytic production of the activator $u$.

(i) Bistable regions

Treating $a$ as the control parameter, eq.(9) in the steady state gives,

$$
\begin{aligned}
& a=b u-k u^{2} v \\
& v=\frac{1}{\left(1+c u^{2}\right)} .
\end{aligned}
$$




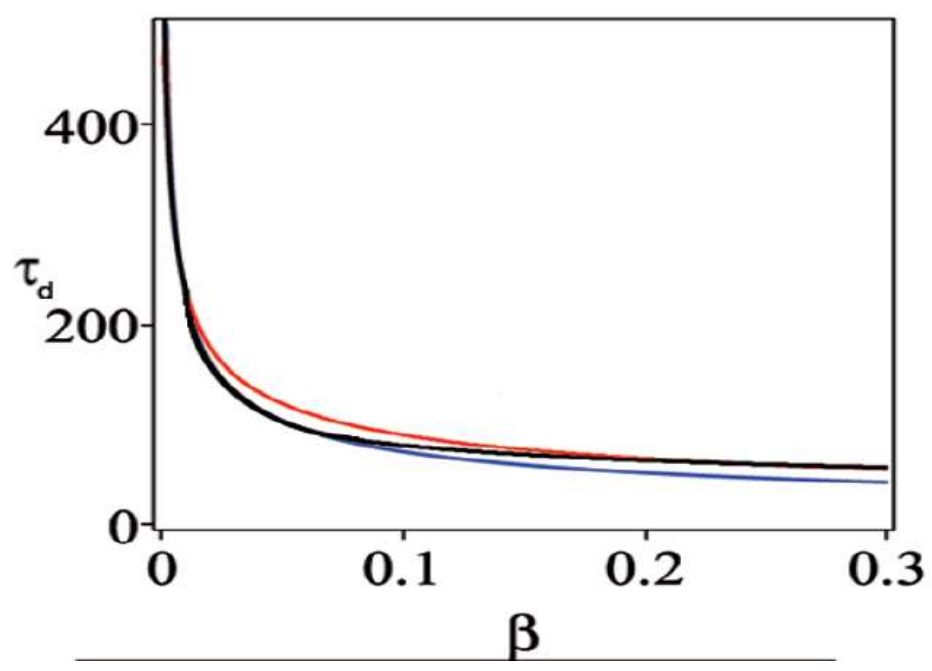

Fig.9: As Fig.4 with black and blue curves for positive and negative perturbations, respectively.
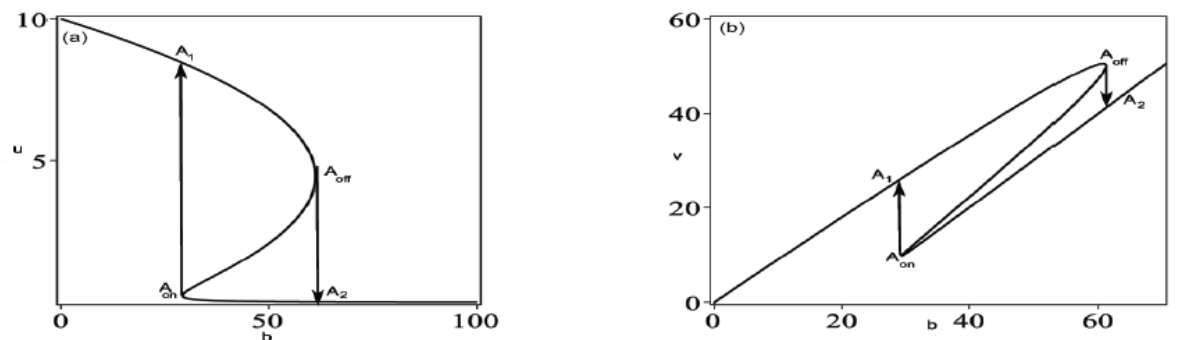

Fig.10: (a) The steady state bistable curve $u$ against $b$, for fixed values of $k=20, a=10, \alpha=0.5, l=10$.

Conditions for bistable behaviour for $u$ and $v$ are determined by the conditions,

$$
\left.S_{5}(b, k) \equiv \frac{d a}{d u}\right|_{u_{i n f}} \leq 0 \text { and }\left.S_{6}(b, k) \equiv \frac{d a}{d v}\right|_{v_{i n f}} \leq 0 .
$$

It can be shown that for fixed $c$ the bistable region for the concentration $u$ exists for $b \leq \frac{9 k}{8 \sqrt{3 c}}$, Fig.11a, while for the concentration $v$ it exists for all $k>0, b>\sqrt{3 c}, \operatorname{Fig} 11(\mathrm{~b})$.

It is noticed that the bistable curve for $v$ is (qualitatively) the mirror 

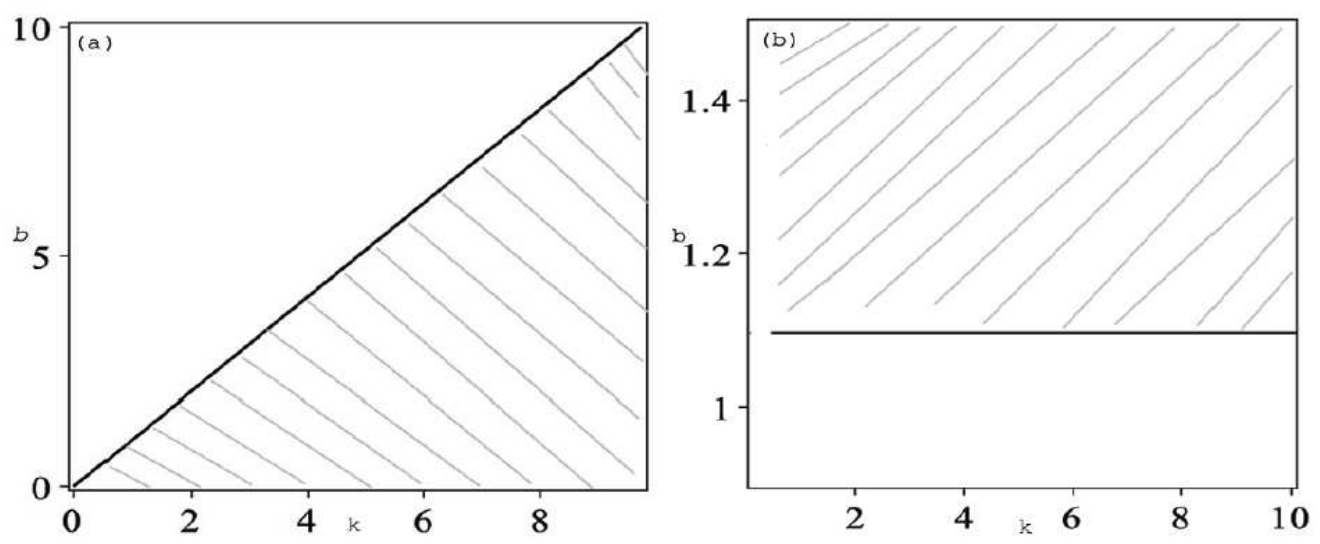

Fig.11: (a) Bistable region for the concentration $u$ in the $(k, b)$-plane with $c=0.4$. (b) As Fig.9(a) but for the concentration $v$.
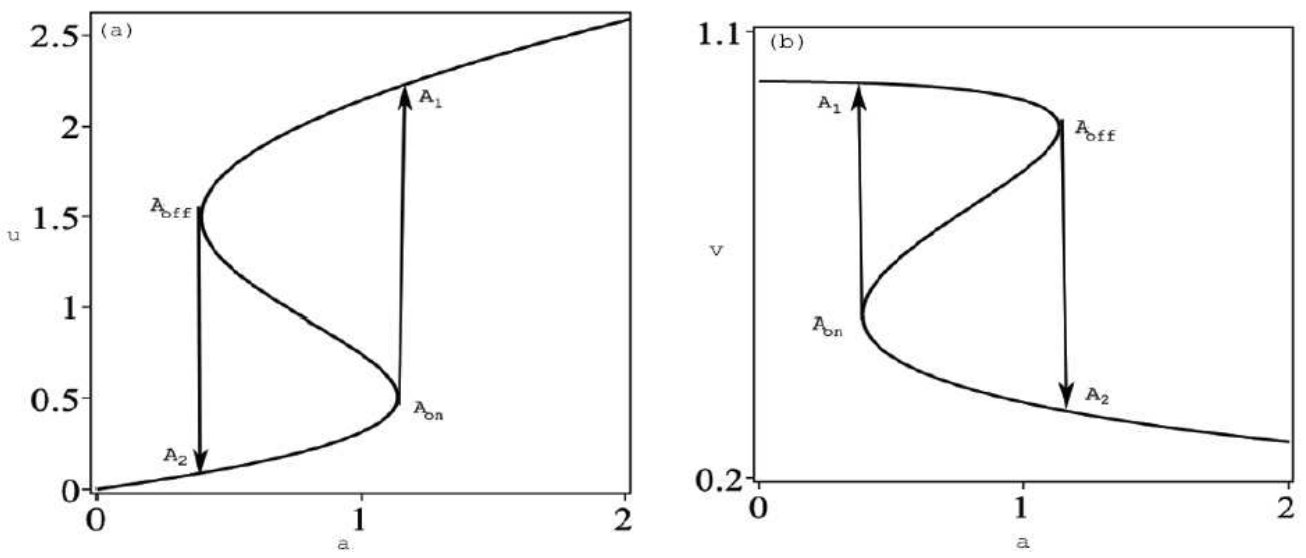

Fig.12: (a)Steady state bistable curve of eq.(10), $u$ against $a$, at fixed values of $k=6, b=5 c=0.4$. The switching-on and -off points: $A_{\text {on }}=(1.1365,0.5066)$ and $A_{o f f}=(0.3947,1.4953)$ with terminal points $A_{1}=(1.1365,2.2141)$ and $A_{2}=(0.3947,0.0883)$, respectively. (b) As (a) but for the concentration $v$ against $a$. The switching-on and -off points: $A_{o n}=(0.3947,0.5278)$ and $A_{o f f}=(1.1365,0.9068)$ with terminal points $A_{1}=(0.3947,0.9968)$ and $A_{2}=(1.1365,0.3377)$, respectively

image of the bistable curve for $u$ (Fig.12). 

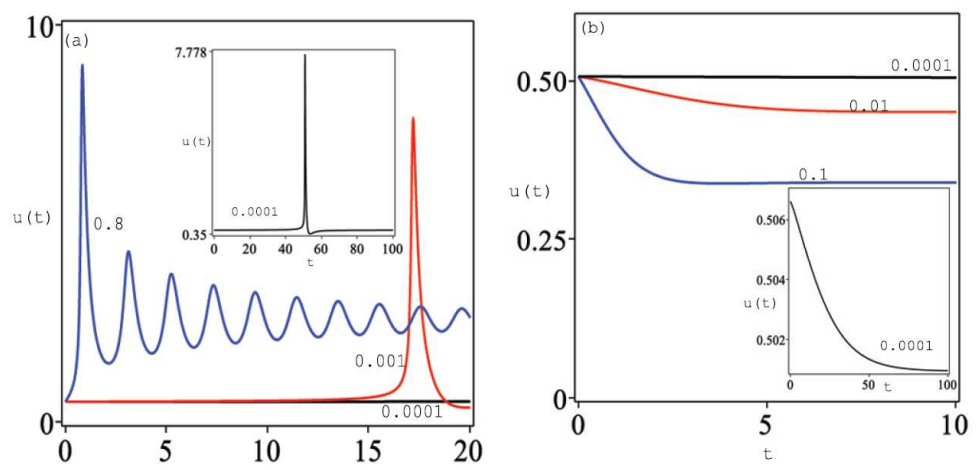

Fig.13: (a) The transient concentration $u(t)$ for $k=6, b=5 c=0.4$ and various values of the control parameter with positive perturbation, $a+\beta$ (after threshold); $a=1.1365$ (at the switching-on point $A_{\text {on }}$ in Fig.5) and $\beta=0.0001,0.01$ and 0.8. Inset shows $u(t)$ for the smallest perturbation $(\beta)$ near the threshold point $A_{\text {on }}$. (b) As (a) but with negative perturbation, $a-\beta$ (before threshold) and $\beta=0.0001,0.01$ and 0.1 .

(ii) Transient switching and delay

The transient switching behaviour at the critical switching-on point $A_{\text {on }}$ in Fig.11(a) for $u(t)$ due to small positive perturbation parameter of the control parameter $a$ is shown in Fig.13(a). There is no effective slowing down effect with changing the perturbation $\beta$ but as $\beta$ increases the activator $u$ approaches its steady state oscillatory. The main reason for this behaviour is the form of the productive term for $u$ due to the nonlinear feedback inhibition, which is mathematically different from the corresponding nonlinear predation and inhibition terms in the spruce budworm infestation and Thomas reaction models, respectively.

For negative perturbation (Fig.13(b)) the activator $u(t)$ switches down monotonically to a very near lower steady state branch, $u: 0.5066 \rightarrow$ 0.5020. The comparison between switching at $A_{\text {on }}$ with positive and negative small perturbation $\beta=10^{-4}$ is shown in Fig.13(c). For the inhibitor $v(t)$, we have similar qualitative, but inverted, graphs as those of Fig.12. The time delay $\tau_{d}$ is shown to fit qualitatively with $\beta^{-\frac{1}{2}}$, independent of the perturbation sign (Fig.14). 


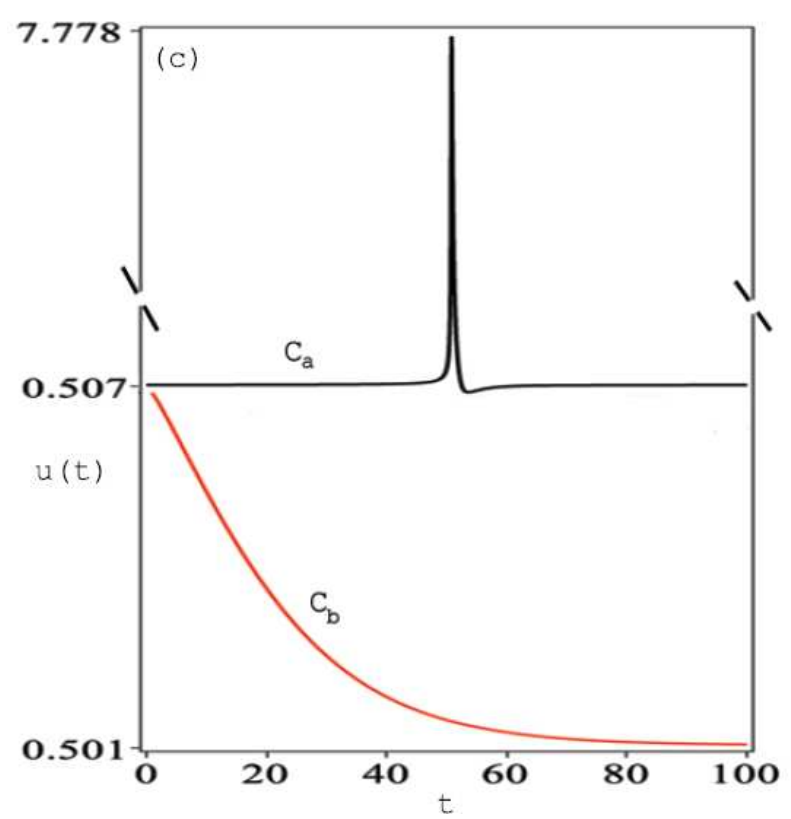

Fig.13: (c) $u(t)$ versus $t$ with positive and negative perturbation, $a \pm \beta$, curves $C_{a}$ and $C_{b}$ (after and before threshold), respectively, with $a=91.1365$ (at the switching-on point $A_{o n}$ in Fig.5) and $\beta=0.0001$.

\section{Summary}

Critical slowing down is a known phenomenon in phase transition theory (e.g. [27] and [28]) where at the critical points of the physical state relation the system relaxation time diverges, and so it switches very slowly (after time delay) to another stable state.

Within the context of bistable phenomena, e.g. in optics and biology, and due to small perturbations in the control (input) parameter near the vicinity of the switching-on and -off points, the system relaxation time to the lower or upper branch of the bistable curve diverges. Study of CSD would benefit (at some degree) the dynamical stabilisation of the system in response to small perturbations or fluctuations at the critical points. With CSD, rates that transients decay to the steady states can be measured. In this work, we have investigated the bistable behaviour and the CSD phenomenon in three specific biological models, namely: the Spruce budworm outbreak model, the Thomas reaction model for enzyme membrane, and Gierer-Meinhardt activator-inhibitor reaction model of biological pattern of tissue formation, with possible control pa- 


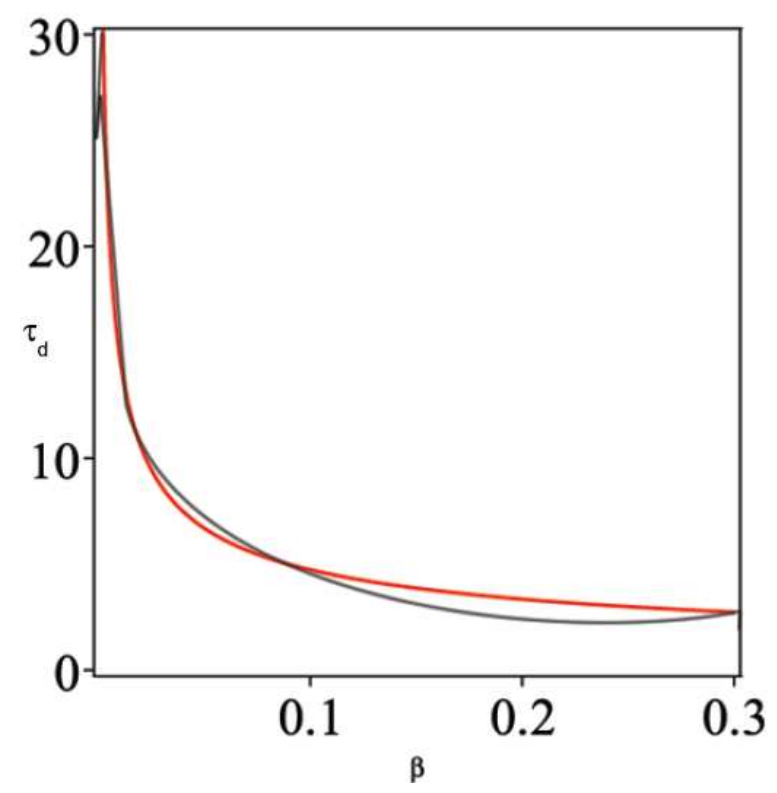

Fig.14: As Fig.4 for the activator-inhibitor model (black curve). The scaling low $\tau_{d}=\tau_{o} \beta^{-\frac{1}{2}} ; \tau_{o}=1.5$ is represented by the red curve.

rameters. Bistable regions were identified computationally and/or analytically according to the respected model rate equations. Depending on the modelled rate equations and the chosen control parameter, the characteristic bistable curves can be the usual (anti-clockwise) S-shape, the inverted S-shape, mirror image and clockwise of the usual S-shape. The switching response at the critical points have been examined computationally. The results show that the time-delay reduction with increasing perturbation parameter to switch sharply or smoothly to the other stable curve is independent on the origin of the nonlinear (feedback, predation, inhibition) terms and fits an inverse square root law for all three models. This is in conformity with experimental [29] and theoretical [29] and [30] investigation for dispersive bistable behaviour in optics, regardless of the origin of the nonlinear dispersion form.

Finally, it is worth referring to the recent analytical work ([31] and refs. therein) which investigates the conditions where CSD can serve as an early indicator of transitions in two-species biological models, that exhibit Hopf and transcritical bifurcations or hysteresis transitions. Our main results here are concerned with the nature of the transient switching process between two stable states and the computational verification that the time-delay reduction near 
the switching points of the bistable curve (CSD) due to perturbed control parameters fits the scaling inverse square root law.

\section{Acknowledgments}

S.S. Hassan acknowledges the hospitality of MMU, School of Computing, Mathematics \& Digital Technology, as a visiting professor (July-August, 2013) where the final version of this joint work was completed. We appreciate fruitful comments from Dr Yasser Sharaby (Suez University, Egypt).

\section{References}

[1] R. Bonifacio and L.A. Lugiato, Cooperative effects and bistability for resonance fluorescence, Opt. Commun., 19, 10.1016/0030-4018(76)903357 (1976), 172-175.

[2] L.A. Lugiato and L.M. Narducci, In: Fundamental Systems in Quantum Optics (Ed-s: J. Dalibard, J. M. Raimond and J. Zinn-Justin), Elsevier Sci. Publ., Amesterdam, (1992), 942-1043.

[3] J.N. Lee (ED), Design Issues in Optical Processing, Cambridge Univ. Press, Cambridge (1997).

[4] S.S. Hassan, P.D. Drummond and D. F. Walls, Dispersive optical bistability in a ring cavity, Opt. Commun., 27, 10.1016/0030-4018(78)904285(1978), 480-484.

[5] S.S. Hassan, H.A. Batarfi, R. Saunders and R.K. Bullough, Bistable behaviour in squeezed vacua: I. Stationary analysis, Eur. Phys. J. D, 8, $10.1007 / \mathrm{s} 100530050051$ (2000), 403-416.

[6] S. Lynch and A.L. Steele, Nonlinear Optical Fibre Resonators with Applications in Electrical Engineering and Computing, in Applications of Nonlinear Dynamics and Chaos in Engineering, Santo Banerjee, Mala Mitra \& Lamberto Rondoni (Eds.), Springer, 1( 2011), 65-84.

[7] Y.A. Sharaby, A. Joshi and S.S. Hassan, Optical bistability without the rotating wave approximation, Phys. Lett. A, 374, 10.1016/j.physleta.2010.03.022 (2010), 2188-2194. 
[8] S.S. Hassan, Y.A. Sharaby, M.F.M. Ali and A. Joshi, Optical bistabilities of higher harmonics: Inhomogeneous and transverse effects, Phys. Lett. A, 376, 10.1016/j.physleta.2012.10.013 (2012), 3555-3561.

[9] A. Joshi and M. Xiao, Optical multistability in three-level atoms inside an optical ring cavity, Phys. Rev. Lett., 91, 10.1103/PhysRevLett.91.143904 (2003), 143904-143907.

[10] A. Joshi, S.S. Hassan, and M. Xiao, Controlling subluminal to superluminal behavior of group velocity with squeezed reservoir, Phys. Rev. A, 72, 10.1103/PhysRevA.72.055803 (2005), 055803-055806.

[11] R. Bonifacio and P. Meystre, Transient response in optical bistability, Opt. Commun., 27, 10.1016/0030-4018(78)90195-5 (1978), 147-150.

[12] S.S. Hassan and Y.A. Sharaby, Critical slowing down in a bistable model with squeezed vacuum environment, Eur. Phys. J. D, 30, 10.1140/epjd/e2004-00107-2 (2004), 393-401.

[13] Y.A. Sharaby, S.S. Hassan and A. Joshi, Critical slowing down with bistable higher harmonics, Opt. Commun., 311, 10.1016/j.optcom.2013.08.041 (2013), 389-396.

[14] P. Mandel and T. Erneux, Nonlinear control in optical bistability, IEEE J. Quantum Electron., QE21 (1985), 1352-1355.

[15] S. Cribier, E. Giacobino, G. Grynberg, Quantitative investigation of critical slowing down in all-optical bistability, Opt. Commun., 47, 10.1016/0030-4018(83)90109-8 (1983), 170-172.

[16] H. A. Al-Attar, Hugh A. MacKenzie, W. J. Firth, Critical slowing-down phenomena in an InSb optically bistable talon, J. Opt. Soc. Am B, 3, 10.1364/JOSAB.3.001157 (1986), 1157-1163.

[17] R.M. May, Thresholds and breakpoints in ecosystems with a multiplicity of stable states, Nature, 269, 10.1038/269471a0 (1977), 471-477.

[18] J.D. Murray, Mathematical Biology: I. An Introduction, 3rd edition, Springer (2002).

[19] S. Lynch and Z. Bandar, Bistable neuromodules, Nonlinear Anal. Theory, Meth. \& Appl., 63, 10.1016/j.na.2004.09.042 (2005), 669-677. 
[20] S. Lynch, Analysis of a blood cell population model, Int. J. of Bifurcation and Chaos, 15, 10.1142/S0218127405013186 (2005), 2311-2316.

[21] I.A. Al-khayat, Bistable behaviour of some models in mathematical biology, In. J. Appl. Maths., 22 (2009), 905-911.

[22] S. Lynch and J. Borresen, Oscillations, feedback and bifurcations in mathematical models of angiogenesis and haematopoiesis, in Handbook of Techniques in Vascular Biology, Slevin M, McDowell G, Cao Y, Kitajewski J (Eds)., Springer, New York, (2013)- (in press).

[23] D. Ludwig, D.D. Jones and C.S. Holling, Qualitative analysis of insect outbreak systems: the spruce budworm and forest, J. Anim. Ecol., 47, $\mathbf{1 0 . 2 3 0 7 / 3 9 3 9 ~ ( 1 9 7 8 ) , ~ 3 1 5 - 3 3 2 . ~}$

[24] D. Thomas, Artificial enzyme membranes, transport, memory and oscillation, In Analysis and Control of Immobilized Enzyme Systems, (Edited by D. Thomas and J. P. Kernevez), Springer (1975), 115-150.

[25] A. Gierer and H. Meinhardt, A theory of biological pattern formation, Kybernetik, 12, 10.1007/BF00289234 (1972), 30-39.

[26] A.C. Fowler, Mathematical models in the applied sciences, Cambridge Univ. Press, Cambridge (1998).

[27] J.M. Yeomans, Statistical mechanics of phase transitions, Oxford, Univ. Press, Oxford (1997).

[28] L.E. Reichl, A modern course in statistical physics, Univ. of Texax Press, USA (1980).

[29] G. Grynberg, F. Biraben, E. Giacobino,Transients in optical bistability, Appl. Phys. B, 26, $10.1007 /$ BF00692440 (1981) 155-160.

[30] G. Grynberg and S. Cribier, Critical exponents in dispersive optical bistability, J. Physicue-Lett. 44, 10.1051/jphyslet:019830044012044900 (1983), L449-L453.

[31] R.A. Chisholm and E. Filotas, Critical slowing down as an indicator of transitions in two-species models, J. Theor. Biol, 257, 10.1016/j.jtbi.2008.11.008 (2009), 142-149. 